\title{
Abstracts of the 37th Annual Meeting of THE JAPAN SOCIETY OF HUMAN GENETICS
}

October 29-31, 1992, Tsukuba, Japan

President: Hideo HAMAGUCHI, M.D.

(Professor, Department of Medical Genetics, Institute of Basic Medical Sciences, University of Tsukuba) 


\section{Plenary Lecture}

PL 1

PRESENT STATUS OF HUMAN GENE THERAPY Takashi SHIMADA (Department of Biochemistry and Molecular Biology, Nippon Medical School, Tokyol

Gene therapy is defined as the treatment of diseases by the transfer of genes into patients cells. This concept was originaliy emerged as the definitive therapy for genetic diseases. However, acquired diseases such as cancer and AIDS are now being considered for its potential targets. Recent advance in retrovirus aediated gene transfer techniques is greatly contributed to the current strategy of gene therapy. Using retroviral vectors, it became possible to introduce genes into many types of cells at the very high efficiency. The first clinical trial of human gene therapy began in 1990 at the NIH for treating a four-year-old girl with severe combined impunodeficiency caused by adenosinedeaminase (ADA) deficiency. A normal ADA gene was introduced into her lymphocytes using a retroviral vector in vitro and gene-corrected and expanded cells were reinfused into ber bloodstreat every four to six weeks. After several infusions, a substantial increase in the number of ADAt cells was observed. The second gene therapy trial started to treat melanoma. Autologous transplantation of tumor infiltrating lymphocytes. (TIL) modified by introducing the tumor necrosis factor (TNF) gene is now underway. In addition, more than 20 Drotocols have already been approved at the recombinant DNA advisory comittee and are about to begin.

\section{Junior Award Lecture}

\section{JA 1}

MEDIUM-CHAIN ACYL-COA DEHYDROGENASE DEFICIENCY: IDENTIFICATION OF MUTATION AND POPULATION SCREENING BY DNA DIAGNOSIS.

\section{Yoichi MATSUBARA}

(Department of Biochemical Genetics, Tohoku University School of Medicine, Sendai)

Medium-chain acyl-CoA dehydrogenase (MCAD) deficiency is an inborn error of fatty acid oxidation metabolism characterized by episodic vomiting, lethargy, and coma. The disorder is potentially fatal and often mis-diagnosed as Reye syndrome or sudden infant death. A point mutation, which substituted Glutamate for Lysine (329) in the precursor MCAD protein, was identified in the MCAD gene as a prevalent mutation among Caucasian patients. The mutation (K329E) was observed in $90 \%$ of mutant MCAD alleles and showed tight linkage with RFLP haplotype 1 of the MCAD gene. Furthermore, population screening using dried blood spot on Guthrie cards obtained from newborns revealed high frequency of K329E carriers in England, Australia, and U.S.A. Several DNA diagnostic methods for the identification of K329E mutation have been developed in our laboratory. Among them, a novel ELISA-based detection system appears to be suitable for processing a large number of samples, enabling mass screening of K329E mutation among Caucasian populations. 